\section{Role of natural drugs in severe acute respiratory syndrome coronavirus 2: potential beneficial effects in prevention or prior treatment in elderly and cancer patients}

\author{
Eugenio Boccalone, ${ }^{1}$ \\ Veronica Maria Lanni, ${ }^{2}$ \\ Valerio Massimo Magro ${ }^{1}$
}

${ }^{1}$ Department of Internal Medicine and

Geriatrics; and ${ }^{2}$ Surgical Sciences

Department, University of Campania

Luigi Vanvitelli, Naples, Italy

In 2019, a new coronavirus, severe acute respiratory syndrome coronavirus 2 (SARS-CoV-2), aroused the attention of the entire world. It causes an acute respiratory disease, by involving the same receptor, i.e. the angiotensin-converting enzyme 2 , as that for severe acute respiratory syndrome coronavirus (SARS-CoV), mainly spreads through the respiratory tract. The clinical symptoms in patients with of SARS-CoV-2 include fever, cough, dyspnea, fatigue and in a small percentage of patients also gastrointestinal symptoms have been reported. The elderly and people with underlying diseases, like cancer patients, are susceptible to infection and prone to serious outcomes, which may be associated with acute respiratory distress syndrome (ARDS). The immune systems of cancer patients are often compromised, weakened by the disease and its treatments, putting them at high risk for the severe form of SARS-CoV-2. In this brief report we focused on the updated literature data about the usefulness in elderly and cancer patients of the natural compounds with antiviral activities against SARS-associated coronavirus, like supportive care, in addition to usual treatments (Figure 1)

The use of natural drugs in the treatment of SARS-CoV-2 was inspired by the treatment protocols previously adopted for SARS-CoV in the late 2002. Several studies examined the role of natural medicines and now there are some evidences about their beneficial effects in the prevention or prior treatment of SARS-CoV. In China, traditional herbal medicines have been frequently used in conjunction with conventional medicine to treat SARS-CoV. Between November 2002 and July 2003, SARSassociated coronavirus caused, in the Asian countries, a pneumonia epidemic. Since this period some studies were made to identify potential natural drugs with antiviral activities against SARS-associated coronavirus. The natural drugs in China have been used in conjunction with conventional medicine to treat SARS-CoV. In a study of $2007,{ }^{1}$ derived from Chinese medicine herbs demonstrated beneficial effects in on the treatment of symptoms of patients with SARS-CoV such as relieving cough, removing the phlegm, and reducing asthma. Glycyrrhizin, an active component of licorice roots, has been reported to show antiviral activity against SARS-CoV in vitro. It was demonstrated that it inhibits the replication of isolates of SARS virus, with a half maximal effective concentration (E50) for the inhibition of viral infection rather high $(E 50=300 \mu \mathrm{g} / \mathrm{mL})$. Another study ${ }^{2}$ identified that lycorine, an active component in the alkaloid portion of the herbal plant Lycoris radiata, shows the best potency $(E 50=2.4 \pm 0.2 \mu \mathrm{g} / \mathrm{mL})$ against SARS$\mathrm{CoV}$. Moreover, lycorine showed a significant anti-tumor activity . Specifically, it has been reported in literature that lycorine demonstrated an anti-tumor activity against human breast cancer cells (MCF-7) and human leukemia cells (HL-60).

Lonicera japonica and Ginsenoside$R b 1$, one of the pharmacologically active compounds of Panax ginseng, were also reported to show inhibition against the SARS-CoV at the concentration of $100 \mu \mathrm{M}$. Resveratrol, a natural compound of grapes, was evaluated ${ }^{3}$ for anti-coronavirus activity in an in vitro cell culture system. It was found that resveratrol completely blocked virus replication at a concentration of 25 $\mu \mathrm{g} / \mathrm{mL}$. The $50 \%$ inhibitory concentration (IC50) was estimated to be $5.5 \mu \mathrm{g} / \mathrm{mL}$. Sambucus nigra extract, characterized largely by quercetin, a flavonoid, was also be reported to be an inhibitor of bovine coronavirus, in vitro. ${ }^{4}$ The inhibition was dose-responsive in that it decreased with decreasing S. nigra extract concentration and increased with decreasing virus concentrations. Treatment with $S$. nigra extracts reduced virus titers by four orders of magnitude. So, the treatment with $S$. nigra extract prior to infection was necessary but not sufficient for full virus inhibition. Additionally, in a paper of 2014, the Authors explained that electronic microscopy of virions treated with $S$. nigra extracts showed compromised envelops and the presence of membrane vesicles. It was found in S. nigra two flavonols $\quad\left(5,7,3^{\prime}, 4^{\prime}\right.$-tetra-O-methylquercetin and 5,7-dihydroxy-4-oxo-2(3,4,5-trihydroxyphenyl) chroman-3-yl3,4,5-trihydroxycyclohexanecarboxylate, which bind to and inhibit influenza virus. Moreover, S. nigra lectins blocked access to
Correspondence: Eugenio Boccalone,

Department of Internal Medicine and Geriatrics, University of Campania Luigi Vanvitelli, piazza L. Miraglia 2, 80100, Naples, Italy.

E-mail: eugeniob.85@gmail.com

Key words: Coronavirus; SARS; elderly patient; medicine herbs.

Contributions: EB and VMM were the primary researchers and wrote the manuscript; $\mathrm{EB}, \mathrm{VML}$ and VMM provided research and editing assistance to the manuscript; VMM provided research and editing assistance to the manuscript. All authors contributed to overall article design, data collection as well as revising and approving the manuscript.

Conflict of interest: the authors declare no potential conflict of interests.

Received for publication: 9 April 2020.

Revision received: 14 April 2020.

Accepted for publication: 14 April 2020.

This work is licensed under a Creative Commons Attribution-NonCommercial 4.0 International License (CC BY-NC 4.0).

${ }^{\circ}$ Copyright: the Author(s), 2020

Licensee PAGEPress, Italy

Geriatric Care 2020; 6:9016

doi:10.4081/gc.2020.9016

host-cell receptors used by infectious bronchitis virus, a pathogenic chicken coronavirus at an early point in infection. All these finding demonstrated that $S$. nigra extract can inhibit a gamma-coronavirus, avian infectious bronchitis virus, at an early phase of the infection and suggested that it does so by compromising virion structure. In a recent clinical study from China, ${ }^{5}$ Authors explained that several patients affected by SARS-CoV-2, with mild symptoms, treated with qingfei paidu decoction (QPD) had several beneficial effects, i.e. the clinical symptom disappearance time was shortened by 2 days, the recovery time of body temperature was shortened by 1.7 days, the average length of stay in hospital was shortened by 2.2 days, the improvement rate of computerized tomography images was increased by $22 \%$, the clinical cure rate was increased by $33 \%, 27.4 \%$ reduction in the rate of common to severe cases and $70 \%$ increase in lymphocyte. Although the results were very interesting, we must note that this study was limited for the absence of any control group and other possible research bias.

In conclusion, natural drugs could be an alternative approach for prevention or early treatment of SARS-CoV-2 in high-risk people, including elderly and cancer patients 


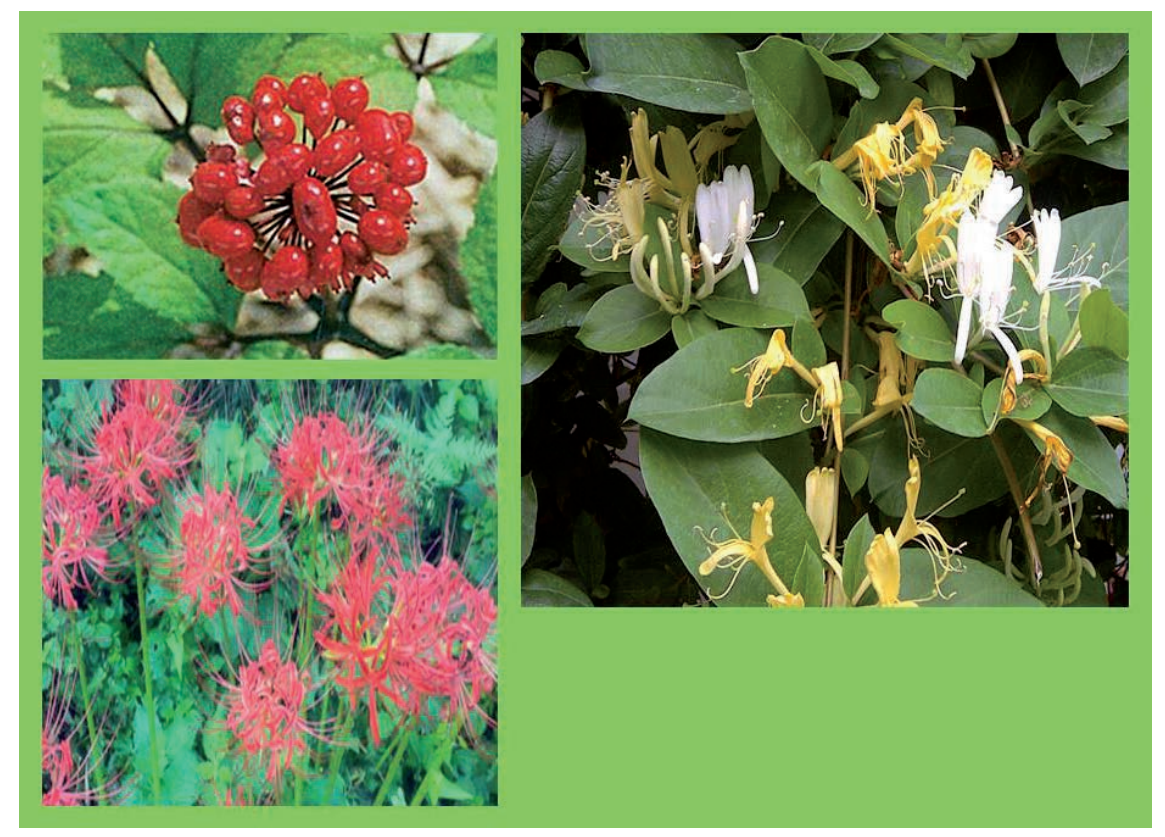

Figure 1. Some of the plants designed to isolate molecules with healing properties towards coronavirus infection: Panax ginseng; Lycoris radiata; Sambucus nigra.

that often cannot use traditional drugs that are toxic for their immune system. Prospective cohort studies with rigorous study design and appropriate sample of patients are need to evaluate the potential role of natural drugs for prevention and cotreatment of SARS-CoV-2 in elderly and cancer patients.

\section{References}

1. Li S-Y, Chen C, Zhang H-Q, et al. Identification of natural compounds with antiviral activities against SARSassociated coronavirus. Antiviral Res 2005;67:18-23.

2. Shen L, Niu J, Wang C, et al. High- throughput screening and identification of potent broad-spectrum inhibitors of coronaviruses. J Virol 2019 [Epub ahead of print].

3. Lin S-C, Ho C-T, Chuo W-H, et al. Effective inhibition of MERS-CoV infection by resveratrol. BMC Infect Dis $2017 ; 17: 144$.

4. Chen C, Zuckerman DM, Brantley S, et al. Sambucus nigra extracts inhibit infectious bronchitis virus at an early point during replication. BMC Vet Res 2014;10:24.

5. Yang Y, Islam MS, Wang J, et al. Traditional Chinese medicine in the treatment of patients infected with 2019-new coronavirus (SARS-CoV-2): a review and perspective. Int $\mathrm{J}$ Biol Sci 2020;16 [Epub ahead of print]. 